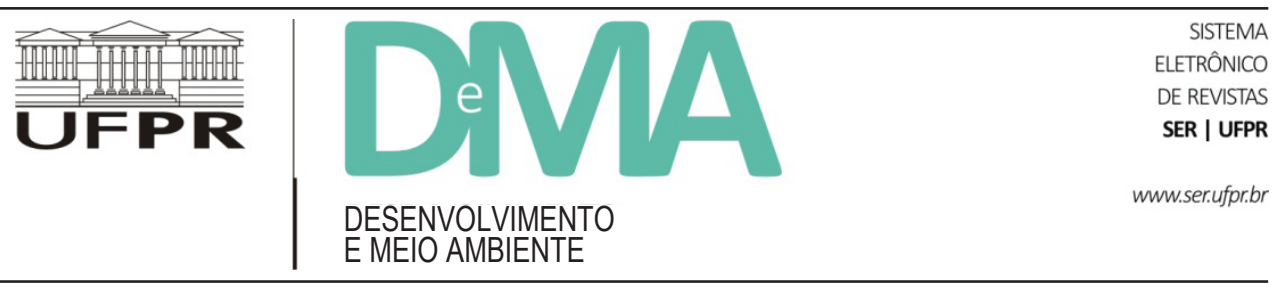

\title{
Variabilidad climática y su impacto en pesquerías de pequeña escala de invertebrados en América Latina*
}

\section{Variabilidade climática e seu impacto em pescarias de pequena escala de invertebrados na América Latina}

\section{Impacts of Climate Variability on Latin American Small-Scale Shellfisheries}

\author{
Omar DEFEO ${ }^{*}$, Mauricio CASTREJÓN ${ }^{2}$, Leonardo ORTEGA³ ${ }^{3}$, Angela M. KUHN², Anita de ÁLAVA ${ }^{1}$ \\ ${ }^{1}$ Universidad de la República (UDELAR), Montevideo, Uruguay. \\ ${ }^{2}$ Dalhousie University, Halifax, Nova Scotia, Canada. \\ ${ }^{3}$ Centro Universitario de la Región Este (CURE), Universidad de la República (UDELAR), Rocha, Uruguay. \\ *E-mail de contacto: odefeo@dinara.gub.uy
}

Artículo recibido el 15 de abril, 2014, versión final aceptada el 18 de julio, 2014.

RESUMEN Las pesquerías de pequeña escala, o artesanales, desempeñan un papel fundamental en la seguridad alimentaria y mitigación de la pobreza en América Latina. Estas pesquerías están cada vez más amenazadas por factores antropogénicos y climáticos que actúan en diversas escalas espacio-temporales. Este trabajo documenta los efectos de la variabilidad climática en pesquerías de pequeña escala de invertebrados en América Latina, basado en literatura reciente. Además se discute el impacto conjunto de dos agentes forzantes adicionales: la globalización de los mercados y la gobernanza. Se ha observado que la variabilidad climática (e.g., anomalías de la temperatura superficial del mar) produce impactos significativos en las pesquerías de pequeña escala, los cuales varían en función del ciclo de vida y la distribución geográfica de las especies objetivo, así como de las características oceanográficas de cada región. Estas variables, en conjunto con factores económicos, han incrementado las tasas de agotamiento de los stocks pesqueros. Se destaca la necesidad de mejorar los sistemas de manejo y gobernanza para hacer frente a la creciente incertidumbre generada por los impactos del cambio climático y la globalización de mercados.

Palabras claves: pesquerías artesanales; invertebrados; variabilidad climática; cambio global; resiliencia; ENSO; América Latina.

\footnotetext{
*Artículo actualizado y traducido al español de la versión publicada en Ecology and Society, 18(4), 30, 2013.
} 
As pescarias de pequena escala ou artesanais desempenham um papel fundamental para a segurança alimentar e para a mitigação da pobreza na América Latina. Estas pescarias estão cada vez mais ameaçadas por fatores antropogênicos e climáticos que atuam em diversas escalas espaço-temporais. Este trabalho documenta os efeitos da variabilidade climática em pescarias de pequena escala de invertebrados na América Latina, com base na literatura recente. Além disso, é discutido o impacto conjunto de duas fontes adicionais: a globalização dos mercados e a governança. Foi observado que a variabilidade climática (e.g., anomalias da temperatura superficial do mar) produz impactos significativos nas pescarias de pequena escala, os quais variam em função do ciclo de vida e da distribuição geográfica das espécies-alvo, assim como nas características oceanográficas de cada região. Estas variáveis, em conjunto com fatores econômicos, têm incrementado as taxas de diminuição dos estoques pesqueiros. É destacada a necessidade de melhorar os sistemas de manejo e governança para lidar com a crescente incerteza causada pelos impactos da mudança climática e da globalização dos mercados.

Palavras-chave: pescarias artesanais; invertebrados; variabilidade climática; mudança global; resiliência; OSEN; América Latina.

ABSTRACT Small-scale fisheries play a vital role in food security and poverty alleviation in Latin America. These fisheries are being increasingly threatened by anthropogenic and climatic factors acting at different spatial and temporal scales. Recent results documenting the effects of climate variability on small-scale shellfisheries in Latin America are reviewed. The joint effects of two additional forcing agents are discussed: the globalization of markets and governance. Significant effects of climate variability (e.g., sea surface temperature anomalies) on small-scale fisheries were observed, which vary according to the life cycle of the species involved, the geographical distribution of the target species and the oceanographic characteristics of each region. Climatic variables, acting in conjunction with economic factors, have increased depletion rates of shellfish stocks. The need to improve governance and management systems to meet the growing uncertainty generated by the impacts of climate change and globalization of markets is highlighted.

Keywords: small-scale fisheries; invertebrates; climate variability; global change; resilience; ENSO; Latin America.

\section{Introducción}

Las pesquerías de pequeña escala o artesanales (SSF por su sigla tradicional en inglés) representan sistemas sociales-ecológicos constituidos por subsistemas biofísicos y sociales que interactúan y se retroalimentan (Ostrom, 2009; Perry et al., 2010; Hall 2011). Las SSF juegan un papel fundamental en los países en desarrollo, tanto en la seguridad alimentaria como en la mitigación de la pobreza (Berkes et al., 2001; Chuenpagdee et al., 2006; Jentoft \& Eide, 2011). Alrededor de 120 millones de personas a nivel mundial viven directa o indirectamente de la actividad pesquera, estimándose que un $90 \%$ de este total participa en pesquerías artesanales, sustentado económicamente a más de 500 millones de personas (FAO, 2012). En América Latina, existen aproximadamente casi 3,000 comunidades pesqueras artesanales y millones de personas directa o indirecta- mente involucrados en esta actividad (Defeo \& Castilla, 2005; Salas et al., 2011). Las SSF se desarrollan en aguas costeras y continentales por pescadores que emplean diferentes artes de pesca para extraer una gran diversidad de recursos (Defeo \& Castilla, 2005). Las herramientas de manejo y los modos de gobernanza que rigen los arreglos institucionales difieren entre pesquerías (Begossi, 2010; Salas et al., 2011).

Las SSF de América Latina están cada vez más amenazadas por factores humanos y climáticos que actúan en múltiples escalas temporales y espaciales, incluyendo el libre acceso, las interdependencias con la pesca industrial y fenómenos climáticos de diversa índole (Bovarnick et al., 2010; Defeo \& Castilla, 2012; Castrejón \& Defeo, en prensa). Estos últimos son especialmente importantes en pesquerías de invertebrados sésiles o sedentarios, los cuales son muy susceptibles a cambios ambientales rápidos debido a su limitada capa- 
cidad de movimiento (Defeo et al., 2009; Heath et al., 2012; Narita et al., 2012; Schoeman et al., 2014). Estos factores afectan a los procesos biofísicos y por lo tanto alteran las poblaciones de invertebrados, incluyendo sus patrones de dispersión, rasgos de su historia de vida y la magnitud de las interacciones interespecíficas (Stenseth et al., 2002; Rouyer et al., 2008; Mellin et al., 2012).

El incremento en el número y la magnitud de los eventos relacionados con el cambio global (Hall, 2011) ha generado la necesidad de comprender la manera en que la variabilidad climática y los efectos antropogénicos están afectando el desempeño de las SSF. A pesar de que el cambio climático ha recibido atención central, existen otros factores que actúan simultáneamente sobre los sistemas pesqueros, cuyo efecto combinado debe ser estudiado (Perry et al., 2010; McCay et al., 2011). En este trabajo se resumen resultados recientes que demuestran los efectos de la variabilidad climática en algunas SSF de invertebrados de América Latina, considerando además efectos adicionales derivados de dos factores humanos: la globalización de mercados y la gobernanza. Se hace énfasis en casos de estudio para los cuales existe información de largo plazo de los subsistemas biofísicos y sociales de las SSF.

\section{Casos de estudio}

\subsection{Las almejas del género Mesodesma}

Un análisis de largo plazo de la macha Mesodesma donacium del Pacífico, especie de origen frío que se distribuye entre $5^{\circ} \mathrm{S}$ y $42^{\circ} \mathrm{S}$, mostró que los eventos cálido del ENSO (El Niño Oscilación del Sur) afectaron negativamente los desembarques en Perú (Figura 1A) y norte de Chile, pero favorecieron los desembarques en el sur de Chile (el extremo meridional de la distribución de la especie), los cuales se correlacionaron positivamente con el aumento de las anomalías en la temperatura superficial del mar (SSTA por su sigla en inglés) (Ortega et al., 2012). Los eventos de El Niño de 1982-83 y 1997 98 fueron particularmente fuertes y se caracterizaron por un aumento significativo de la temperatura del mar (Ortega et al., 2012), causando mortandades masivas de M. donacium (Arntz et al., 1987; Riascos et al., 2009).
Otra respuesta diferencial a los eventos climáticos extremos se observó para el ostión o concha de abanico Argopecten purpuratus explotada artesanalmente en el norte de Perú: los eventos severos de El Niño aumentaron drásticamente las inundaciones y descargas de los ríos, provocando una disminución en la biomasa del ostión, mientras que el aumento de las temperaturas en el Sur produjo un aumento de su población (Badjeck et al., 2009). Durante el evento de El Niño 1997-98, la SST en la Bahía de la Independencia ( $14^{\circ} \mathrm{S}$, Perú) aumentó $10^{\circ} \mathrm{C}$ y se registraron altas concentraciones de oxígeno y concentraciones reducidas de fitoplancton. Varias especies bentónicas se vieron afectadas (e.g., macroalgas, cangrejos y poliquetos), pero la biomasa de $A$. purpuratus aumentó un 50\% (Taylor et al. 2008). De forma similar, Defeo \& Castilla (1998) describieron un aumento exponencial en la abundancia y desembarques del pulpo Octopus mimus en el norte de Chile, durante y después de El Niño de 1982-83. El aumento de la temperatura mejoró su reclutamiento y la disponibilidad de presas (Castilla \& Camus, 1992).

En el Océano Atlántico Sudoccidental, la abundancia de la almeja amarilla Mesodesma mactroides (especie de origen frío) disminuyó en el largo plazo por un efecto combinado de aumento de las SSTA y de la intensidad de pesca (Ortega et al., 2012). La abundancia de la almeja amarilla estuvo inversamente correlacionada con el aumento de las SSTA (Figura 1B), sugiriendo una mayor abundancia en períodos fríos. Las mortandades masivas de $M$. mactroides que ocurrieron de forma secuencial en una dirección norte-sur desde 1993 (Sur de Brasil) a 2002 (Argentina), coincidieron con el cambio de la Oscilación Multidecadal del Atlántico (AMO, por sus siglas en inglés) de un periodo frío a uno cálido a partir de 1994 (Goldenberg et al., 2001). Estas mortandades se dieron principalmente entre fines de primavera y principios del verano, cuando estas almejas son más susceptibles a contraer enfermedades (Fiori et al., 2004; Herrmann et al., 2011). El aumento sistemático de las SSTA ha exacerbado la influencia negativa de aguas cálidas en las poblaciones de almeja (Ortega et al., 2012; Defeo et al., 2013).

Los efectos de la variabilidad climática han superado largamente el impacto de las medidas de manejo implementadas para recuperar a las poblaciones. En Perú, 


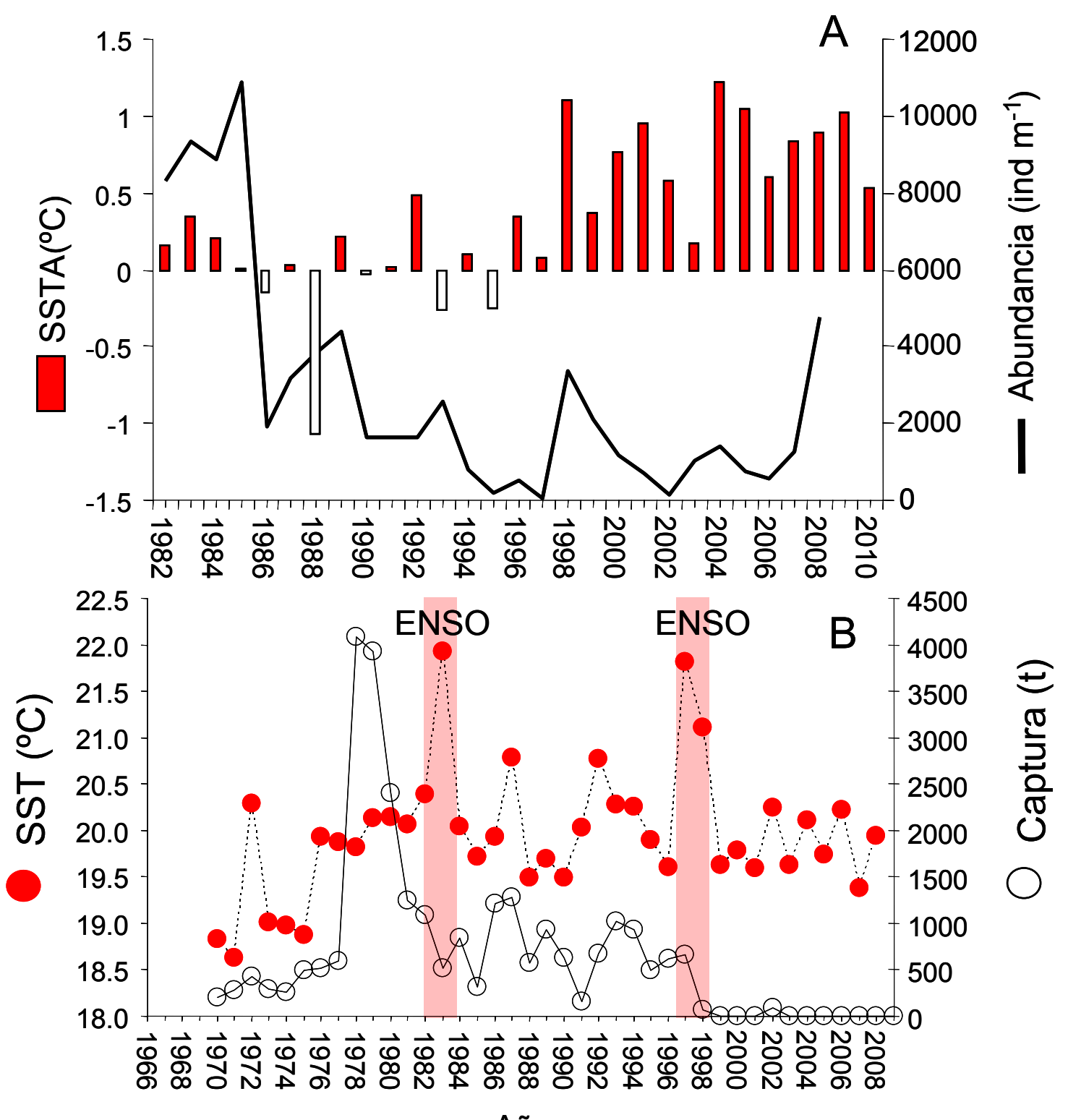

Año

FIGURA 1 - Variaciones de largo plazo en: A) las anomalías de temperatura superficial del agua de mar (SSTA) y en la abundancia de la almeja amarilla Mesodesma mactroides en Uruguay; y B) la temperatura superficial del agua de mar (SST) y las capturas de la macha Mesodesma donacium en Perú, resaltándose los dos ENSO fuertes que ocurrieron en 1982-1983 y 1997-1998. 
los efectos adversos del clima en la macha $M$. donacium potenciaron los impactos negativos de los niveles de esfuerzo pesquero insostenibles y el acceso abierto al recurso. En efecto, después del fenómeno de El Niño de 1997-98, la macha casi desapareció y la pesquería fue cerrada en 1999 (Defeo et al., 2013). La especie no se ha recuperado en los últimos 16 años, lo cual ha motivado que la pesquería siga cerrada hasta el presente (Ortega et al., 2012). Similares efectos negativos en la demografía de la macha han sido documentados por Aburto \& Stotz (2013) en el norte de Chile. En las costas del Atlántico, las mortandades masivas de almeja amarilla M. mactroides determinaron el cierre de la pesquería en Uruguay y Argentina por casi dos décadas (Fiori \& Defeo, 2006). La falta de respuesta de los stocks a estas clausuras pesqueras sugiere que estos sistemas podrían haber superado umbrales críticos (Scheffer et al., 2009), lo cual ha producido una transición a otro estado que incluye variaciones drásticas en la composición de la comunidad (Defeo et al., 2013 y referencias contenidas en éste).

\subsection{Las pesquerías de langosta espinosa y pepino de mar de Galápagos}

Las Islas Galápagos (Ecuador) representan un sistema único para evaluar el impacto de la variabilidad climática sobre las especies marinas, debido a su ubicación en el Océano Pacífico Ecuatorial, la principal zona de influencia del ENSO. Análisis de largo plazo (Edgar et al., 2010) han mostrado que los hábitats poco profundos de la zona central del archipiélago experimentaron grandes transformaciones durante el evento de El Niño 1982-83, incluyendo una disminución marcada en la biodiversidad. De forma similar, Wolff et al. (2012a) mostraron que la SST se incrementó en $7^{\circ} \mathrm{C}$ durante el El Niño de 1997-98, disminuyendo la concentración de fitoplancton y el tamaño del ecosistema, reflejándose en una merma de la abundancia de peces, aves, reptiles y mamíferos marinos.

No todas las especies se vieron afectadas negativamente por lo dichos eventos cálidos: las biomasas de langostas espinosas (Panulirus gracilis y P. penicillatus) y de pepinos de mar (Isostichopus fuscus) aumentaron después de El Niño de 1997-98. Éste representó el evento climático más intenso registrado en los últimos 30 años. El incremento de biomasa repercutió en los desembarques de estos invertebrados. Dos y cinco años después de dicho evento, se registró un incremento máximo histórico en la producción de langosta (Figura 2A) y pepino (Figura 2B), respectivamente (ver además Defeo et al., 2013). Este aumento estuvo influenciado además, en ambos casos, por un incremento en el esfuerzo de pesca (Hearn et al., 2005; Castrejón, 2011; Wolff et al., 2012a). Esto sugiere que el aumento de SST favoreció un incremento de la abundancia de ambos recursos y el desarrollo de la pesquería (Defeo et al., 2013).
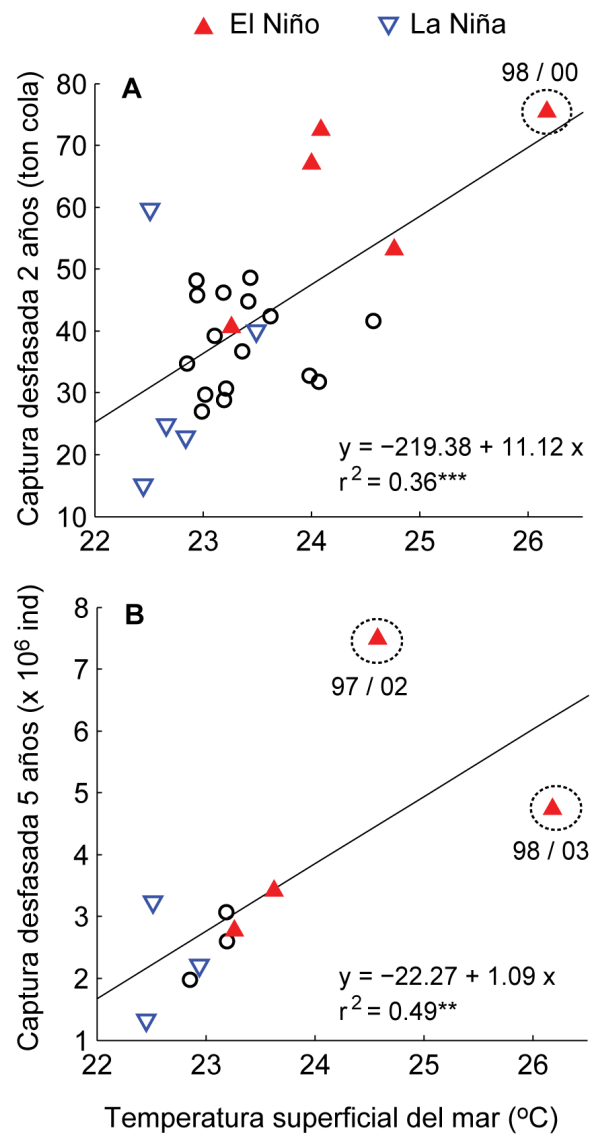

FIGURA 2 - Variaciones de largo plazo en la relación entre la temperatura superficial del agua de mar y las capturas de langosta espinosa (A) y pepino de mar (B) en Galápagos, desfasadas en el tiempo. Se distinguen los períodos de La Niña y El Niño y se remarcan los severos eventos de El Niño con círculos punteados. 
Otros efectos ecosistémicos en cascada, tales como una menor abundancia de depredadores (e.g., peces demersales) y una alta abundancia de presas (e.g., erizos de mar) luego de El Niño de 1997-98, podrían explicar la alta producción de langostas espinosas en ese período (Bustamante et al., 2002; Hearn \& Murillo, 2008; Wolff et al., 2012b). El efecto combinado de un reclutamiento deficiente y altos niveles de pesca afectó severamente la pesquería de pepino, razón por la cual ha sido clausurada en cinco ocasiones desde 2006.

\section{Discusión}

Los estudios de largo plazo en SSF de invertebrados de Latinoamérica revisados en este trabajo sugieren efectos importantes de la variabilidad climática y de agentes económicos y de gobernanza de Latinoamérica. El impacto de los diferentes factores varía en función del ciclo de vida de las especies, las variables oceanográficas y las características inherentes de los sistemas sociales. Los factores climáticos y antropogénicos interactúan y alteran estos sistemas sociales-ecológicos en forma no lineal, magnificando las respuestas de los ecosistemas al cambio climático y limitando su capacidad de predicción y adaptación (Ling et al., 2009, Perry et al., 2010; 2011). Por tanto, es difícil aislar los factores naturales de los provocados por el hombre, debido en parte a la incertidumbre en la magnitud de impactos específicos sobre varios componentes del ecosistema (Grafton, 2010; Barange et al., 2010). Dado que la magnitud de las capturas ha cambiado en forma asociada con las tendencias de calentamiento global (Cheung et al., 2013), la necesidad de tener en cuenta las condiciones ambientales al formular estrategias de gestión ha adquirido más importancia que nunca.

El ciclo de vida de los invertebrados debe ser considerado a efectos de un adecuado manejo (Orensanz \& Jamieson, 1998). Las grandes fluctuaciones en la abundancia de este tipo de organismos, en parte explicables por factores climáticos, han llevado a describir sus poblaciones como "resurgentes" (McLachlan et al., 1996). El reclutamiento exitoso está por lo general vinculado a condiciones climáticas favorables (e.g., variaciones de SST), lo cual genera estructuras poblacionales do- minadas por pocas clases de edad. Teniendo en cuenta que muchos de estos recursos se estructuran como metapoblaciones, definidas por una larva planctónica y una fase adulta bentónica, el reclutamiento tiende a ocurrir con regularidad en hábitats fuente y a ser irregular o espasmódico en hábitats receptores de menor calidad (Caddy \& Defeo, 2003). Esto genera a su vez una alta variabilidad en las pesquerías, pasando por un ciclo de "auge y caída" con desembarques inicialmente altos en concurrencia con reclutamientos exitosos, que luego disminuyen cuando las situaciones climáticas desfavorables conducen a un reclutamiento pobre. Una respuesta desfavorable de las especies y del hábitat a agentes climáticos puede determinar la reducción en el número de días de pesca y en los retornos económicos resultantes de la actividad (Defeo et al., 2013). Por tanto, el manejo de las pesquerías de invertebrados debe considerar el efecto de estas variaciones ambientales en la abundancia y en la calidad del hábitat (Orensanz \& Jamieson, 1998; Caddy, 2007; Defeo et al., 2013). Esto resalta la necesidad de aplicar la teoría de metapoblaciones para el manejo de las pesquerías de invertebrados en el contexto de la variabilidad climática.

El reconocimiento de patrones espaciales en la dinámica y demografía de la poblaciones de invertebrados es de importancia para el manejo de las pesquerías (Defeo \& Castilla, 2005; Orensanz et al., 2005; Castrejón \& Charles, 2013). Por tanto, resulta crítico unificar las escalas espaciales relacionadas con los ciclos de vida de las poblaciones y las unidades funcionales de manejo (Castilla \& Defeo, 2001; Orensanz et al., 2005; McCay \& Jones, 2011; White \& Costello, 2011). Si se cumple este requisito, una combinación de herramientas espacialmente explícitas que incluya la asignación de derechos de uso territorial (TURFs) y Áreas Marinas Protegidas (AMPs) estratégicamente localizadas, podría incrementar tanto los beneficios ecológicos como económicos (Costello \& Kaffine, 2009) y, al mismo tiempo, podría responderse a eventos climáticos desfavorables con mayor eficacia (McCay \& Jones, 2011). Micheli et al. (2012) mostraron que, a pesar de los eventos de mortandades masivas y generalizadas de invertebrados bentónicos en Baja California (México), el reclutamiento de los juveniles de abalón rosado Haliotis corrugata permaneció estable dentro de las AMPs, debido a la alta 
producción de huevos de adultos con tallas grandes. Estas herramientas podrían ser complementadas por cuotas de captura y por otras medidas operacionales de manejo, en un marco de redundancia (Gutiérrez et al., 2011).

La vulnerabilidad de una especie al cambio climático depende de su exposición y susceptibilidad a la variabilidad del clima, su resiliencia frente a las perturbaciones y su potencial para adaptarse al cambio (Williams et al., 2008; Mumby et al., 2014). Estos criterios de vulnerabilidad requieren información científica sólida sobre datos conductuales, fisiológicos y genéticos (Huey et al., 2012; Doney et al., 2012), los cuales escasean en especies objetivo de las SSF en América Latina. Asimismo, la evaluación de la vulnerabilidad al cambio climático requiere de un esfuerzo multidisciplinario para desarrollar marcos de gestión adaptativos, dirigidos a mitigar los efectos de los factores climáticos en las especies y en el bienestar de las comunidades costeras (Grafton, 2010; Cinner et al., 2012b), Lamentablemente, los organismos reguladores de la pesca en América Latina, que en general poseen un escaso presupuesto para actividades de investigación, monitoreo, control y vigilancia, tienden a responder con retraso a los problemas que se suscitan, una vez que son más difíciles o incluso imposibles de resolver. Esta situación se agrava por la carencia de estrategias de planificación y de políticas de estado (Defeo \& Castilla, 2012). Como las SSF tienden a estar fuertemente influenciadas por intereses económicos y políticos de corto plazo, son menos resistentes y más vulnerables a los desafíos a largo plazo asociados con la variabilidad climática (Jentoft \& Chuenpagdee, 2009). La sobrepesca reduce la resiliencia de las poblaciones a los cambios inducidos por fases catastróficas del clima (Ling et al., 2009), por lo cual la detección de señales de alerta temprana es de importancia crítica (Scheffer et al., 2009; Lenton, 2011). No obstante, esto dista mucho de hacerse realidad en América Latina.

La gobernanza de las pesquerías costeras de invertebrados en América Latina puede verse fortalecida a través de soluciones escala-dependientes. A escala local, algunas soluciones incluyen el desarrollo de esquemas con la asignación de derechos de uso territorial y la adopción del co-manejo como modo de gobernanza (Basurto, 2005; Defeo \& Castilla, 2005; 2012; Hilborn et al., 2005; Gelcich et al., 2010). El co-manejo adaptativo en comunidades locales con una fuerte organización y cohesión interna permite desarrollar mecanismos para hacer frente a la influencia de la variabilidad climática en la abundancia y disponibilidad de los recursos (Kalikoski et al., 2010; Castrejón \& Defeo, en prensa), fomentando respuestas de adaptación flexibles y fortaleciendo las capacidades adaptativas a los diferentes factores (Grafton, 2010; Cinner et al., 2012b). A mayor escala, la zonificación del mar para las flotas artesanales e industriales, incluyendo la asignación de los derechos exclusivos espaciales a las comunidades artesanales, ha mitigado los problemas de gobernanza en algunos países como Chile (Castilla, 2010) y Uruguay (Horta \& Defeo, 2012). Sin embargo, es necesario generar vínculos estrechos entre las instituciones locales con aquellas situadas a niveles más altos de gobierno (Cinner et al., 2012a). Esto tiene importancia adicional en recursos transfronterizos con alto valor comercial (e.g., langostas espinosas), que requieren de convenios regionales para ser adecuadamente gestionados. Todo lo anterior resalta la necesidad de reconocer y apoyar formalmente esquemas de gobernanza transversales que incluyan la institucionalización de los derechos pesqueros en SSF de Latinoamérica (Defeo \& Castilla, 2005; Chakalall et al., 2007).

A los factores climáticos y de gobernanza antes señalados, se le agrega un agente forzante generado por la globalización de los mercados. El déficit de oferta e incremento de la demanda procedente de países desarrollados donde invertebrados de gran importancia comercial han sido previamente sobreexplotados (e.g., pepino de mar), ha potenciado un aumento sostenido del precio unitario de este tipo de recursos en América latina. Esto ha provocado un aumento exponencial en el esfuerzo de pesca (incluso bajo tasas de captura decrecientes) debido a los bajos costos de inversión y operación, lo cual ha resultado en el colapso económico secuencial de varias SSF de invertebrados de América Latina (Defeo \& Castilla, 2012; Defeo et al., 2013). Asimismo, la presencia de agentes mercantilistas externos ha promovido la extracción ilegal de recursos en muchas pesquerías de invertebrados con alto valor unitario (Defeo \& Castilla, 2012). Dichos agentes han estimulado la extracción ilegal al ofrecer altos precios de comercialización a comunidades locales escasas en recursos económicos y con limitadas o nula fuentes de empleo alternativo (Cas- 
trejón \& Defeo, en prensa). En este contexto, el comercio ilegal aceleró las tasas de agotamiento de los recursos, aprovechando las altas preferencias intertemporales en su uso y una insuficiente aplicación e ineficaz fiscalización de las medidas de manejo (Defeo \& Castilla, 2012). Por ejemplo, aún en el caso de efectos positivos del clima, como en la pesquería de pepino de mar de Galápagos, la débil gobernanza facilitó la extracción ilegal que llevó a su sobreexplotación en el corto plazo (Castrejón, 2011). Esto resalta la necesidad de desarrollar sólidos sistemas de gobernanza de la pesca a través de la consolidación de instituciones fuertes que promuevan la resiliencia bajo escenarios de incertidumbre en los aspectos biofísicos y sociales (Gutiérrez et al., 2011).

En suma, el manejo de las SSF de invertebrados en América Latina debe considerar las interacciones entre los factores que afectan los subsistemas biofísicos y sociales. En este contexto, la débil gobernanza, conjuntamente con la erosión de los sistemas tradicionales de uso de los recursos, el libre acceso, la pobreza, la falta

\section{Referencias}

Aburto, J.; Stotz, W. Learning about TURFs and natural variability: Failure of surf clam management in Chile. Ocean and Coastal Management, 71, 88-98, 2013.

Arntz, W. E.; Brey, T.; Tarazona, J.; Robles, A. Changes in the structure of a shallow sandy-beach community in Peru during an El Niño event. En: Payne, A. I.; Gulland, J. A.; Bink, K. H. (Eds.). The Benguela and comparable ecosystems. South African Journal of Marine Science, 5, 645-658, 1987.

Badjeck, M. C.; Mendo, J.; Wolff, M.; Lange, H. Climate variability and the Peruvian scallop fishery: the role of formal institutions in resilience building. Climatic Change, 94, 211232, 2009.

Barange, M.; Cheung, W. W. L.; Merino, G.; Perry, R. I. Modelling the potential impacts of climate change and human activities on the sustainability of marine resources. Current Opinion in Environmental Sustainability, 2, 326-333, 2010.

Basurto, X. How locally designed access and use controls can prevent the tragedy of the commons in a Mexican small-scale fishing community. Society and Natural Resources, 18, 643-659, 2005. de empleo alternativo y el fácil acceso a los stocks con bajos costos operativos, ha promovido la sobrepesca, socavado los sistemas de gobernanza y aumentado la vulnerabilidad de las comunidades al cambio climático. Las instituciones de gobierno han sido incapaces de adoptar acciones proactivas y efectivas para lidiar con el impacto combinado de la pesca y la variabilidad climática en el bienestar de las comunidades. Por tanto, para hacer frente a la creciente incertidumbre generada por el efecto de múltiples agentes externos, se necesitan instituciones sólidas y normas de manejo efectivas que garanticen SSF sustentables en América Latina.

\section{Agradecimientos}

Se agradece el apoyo financiero proporcionado por el Fondo Global para el Medio Ambiente (GCP URU 030 GFF), el Programa de Desarrollo de las Ciencias Básicas y The Pew Charitable Trusts.
Begossi, A. Small-scale fisheries in Latin America: management models and challenges. Maritime Studies, 9, 7-31, 2010.

Berkes, F.; Mahon, R.; McConney, P.; Pollnac, R.; Pomeroy, R. Managing small-scale fisheries. Alternative directions and methods. International Development Research Centre, Ottawa, Canada, 2001.

Bovarnick, A.; Alpizar, F.; Schnell, C. (Eds.). The importance of biodiversity and ecosystems in economic growth and equity in Latin America and the Caribbean: an economic valuation of ecosystems. United Nations Development Programme, 2010.

Bustamante, R. H.; Branch, G. M.; Bensted-Smith, R.; Edgar, G. J. The status of and threats to marine biodiversity. In: Bensted-Smith, R. (Ed.) A biodiversity vision for the Galapagos Islands. Charles Darwin Foundation and World Wildlife Fund, Galápagos, Ecuador, p. 80-95, 2002.

Caddy, J. F. Marine habitat and cover: their importance for productive coastal fishery resources. UNESCO Publishing, Paris, France, 2007.

Caddy, J. F.; Defeo, O. Enhancing or restoring the productivity of natural populations of shellfish and other marine inverte- 
brate resources. FAO Fisheries Technical Paper $\mathrm{N}^{\circ} 448, \mathrm{FAO}$, Rome, 2003.

Castilla, J. C. Fisheries in Chile: small pelagics, management, rights, and sea zoning. Bulletin of Marine Science, 86, 221234, 2010.

Castilla, J. C.; Camus, P. A. The Humboldt El Niño Scenario: coastal benthic resources and anthropogenic influences, with particular reference to the 1982/83 ENSO. South African Journal of Marine Science, 12, 703-712, 1992.

Castilla, J. C.; Defeo, O. Latin-American benthic shellfisheries: emphasis on co-management and experimental practices. Reviews in Fish Biology and Fisheries, 11, 1-30, 2001.

Castrejón, M. Co-manejo pesquero en la Reserva Marina de Galápagos: tendencias, retos y perspectivas de cambio. Fundación Charles Darwin and Kanankil/Plaza Valdés, México, 2011.

Castrejón, M.; Charles, T. Improving fisheries co-management through ecosystem-based spatial management: The Galapagos Marine Reserve. Marine Policy, 38, 235-245, 2013.

Castrejón, M.; Defeo, O. Co-governance of small-scale shellfisheries in Latin America: Institutional capacity to cope with external drivers of change. In: Jentoft, S.; Chuenpagdee, R. (Eds.) Governing the Governance of Small-Scale Fisheries. MARE Series, Springer: en prensa.

Chakalall, B.; Mahon, R.; McConney, P.; Nurse, L.; Oderson, D. Governance of fisheries and other living marine resources in the Wider Caribbean. Fisheries Research, 87, 92-99, 2007.

Cheung, W. W. L.; Watson, R.; Pauly, D. Signature of ocean warming in global fisheries catch. Nature, 497, 365-369, 2013.

Chuenpagdee, R.; Liguori, L.; Palomares, M. L. D.; Pauly, D. Bottom-up, global estimates of small-scale fisheries catches. Fisheries Centre Research Report 14, Vancouver, Canada, 2006.

Cinner, J. E.; Basurto, X.; Fidelman, P.; Kuange, J.; Lahari, R.; Mukminin, A. Institutional designs of customary fisheries management arrangements in Indonesia, Papua New Guinea, and Mexico. Marine Policy, 36, 278-285, 2012a.

Cinner, J. E.; McClanahan, T. R.; Graham, N. A. J.; Dawc, T. M.; Maina, J.; Stead, S. M.; Wamukota, A.; Brown, K.; Bodin, O. Vulnerability of coastal communities to key impacts of climate change on coral reef fisheries. Global Environmental Change, 22, 12-20, 2012b.

Costello, C.; Kaffine, D. T. Marine protected areas in spatial property-rights fisheries. The Australian Journal of Agricultural and Resource Economics, 54, 321-341, 2009.
Defeo, O.; Castilla, J. C. Harvesting and economic patterns in the artisanal Octopus mimus (Cephalopoda) fishery in a northern Chile cove. Fisheries Research, 38, 121-130, 1998.

Defeo, O.; Castilla, J. C. More than one bag for the world fishery crisis and keys for co-management successes in selected artisanal Latin American shellfisheries. Reviews in Fish Biology and Fisheries, 15, 265-283, 2005.

Defeo, O.; Castilla, J. C. Governance and governability of coastal shellfisheries in Latin America and the Caribbean: multi-scale emerging models and effects of globalization and climate change. Current Opinion in Environmental Sustainability, 4, 344-350, 2012.

Defeo, O.; Castrejón, M.; Ortega, L.; Kuhn, A.; Gutiérrez, N. L.; Castilla, J. C. Impacts of climate variability on Latin American small-scale fisheries. Ecology and Society, 18(4), 30, 2013.

Defeo, O.; McLachlan, A.; Schoeman, D. S.; Schlacher, T.; Dugan, J.; Jones, A.; Lastra, M.; Scapini, D. F. Threats to sandy beach ecosystems: a review. Estuarine, Coastal and Shelf Science, 81, 1-12, 2009.

Doney, S. C.; Ruckelshaus, M.; Duffy, J. E.; Barry, J. P.; Chan, F.; English, C. A.; Galindo, H. M.; Grebmeier J. M.; Hollowed, A. B.; Knowlton, N.; Polovina, J.; Rabalais, N. N.; Sydeman, W. J.; Talley, L. D. Climate change impacts on marine ecosystems. Annual Review of Marine Science, 4, 11-37, 2012.

Edgar, G. J.; Banks, S. A.; Brandt, M.; Bustamante, R. H.; Chiriboga, A.; Earle, S. A.; Garske, L. E.; Glynn, P. W.; Grove, J. S.; Henderson, S.; Hickman, C. P.; Miller, K. A.; Rivera, F.; Wellington, G. M. El Niño, grazers and fisheries interact to greatly elevate extinction risk for Galapagos marine species. Global Change Biology, 16, 2876-2890, 2010.

FAO. Report of the Workshop on International Guidelines for Securing Sustainable Small-Scale Fisheries. FAO Fisheries and Aquaculture Report No 1004, Rome, 2012.

Fiori, S.; Defeo, O. Biogeographic patterns in life- history traits of the yellow clam, Mesodesma mactroides, in sandy beaches of South America. Journal of Coastal Research, 22, 872-880, 2006.

Fiori, S.; Vidal-Martínez, V.; Simá-Álvarez, R.; Rodríguez-Canul, R. M.; Aguirre-Macedo, L.; Defeo, O. Field and laboratory observations of the mass mortality of the yellow clam Mesodesma mactroides in South America: the case of Isla del Jabalí, Argentina. Journal of Shellfish Research, 23, 451-455, 2004.

Gelcich, S.; Hughes, T.; Olsson, P.; Folke, C.; Defeo, O.; Fernández, M.; Foale, S.; Gunderson, L.; Rodríguez-Sickert, 
C.; Scheffer, M.; Steneck, R.; Castilla, J. C. Navigating transformations in governance of Chilean marine coastal resources. Proceedings of the National Academy of Sciences USA, 107, 16794-16799, 2010.

Goldenberg, S. B.; Landsea, C. W.; Mestas-Nuñez, A. M.; Gray, W. M. The recent increase in Atlantic hurricane activity: causes \& implications. Science, 293, 474-479, 2001.

Grafton, R. Q. Adaptation to climate change in marine capture fisheries. Marine Policy, 34, 606-615, 2010.

Gutiérrez, N. L.; Hilborn, R.; Defeo, O. Leadership, social capital and incentives promote successful fisheries. Nature, 470, 386-389, 2011.

Hall, S. J. Climate change and other external drivers in small-scale fisheries: practical steps for responding. In: Pomeroy, R. S.; Andrew, N. L. (Eds.) Small-scale fisheries management: Frameworks and approaches for the developing world. $\mathrm{CAB}$ International, Cambridge, 2011. p. 132-159.

Hearn, A.; Martínez, P.; Toral-Granda, M. V.; Murillo, J. C.; Polovina, J. Population dynamics of the exploited sea cucumber Isostichopus fuscus in the Western Galapagos Islands, Ecuador. Fisheries Oceanography, 14, 1-9, 2005.

Hearn, A.; Murillo, J. C. Life history of the red spiny lobster, Panulirus penicillatus (Decapoda: Palinuridae), in the Galapagos Marine Reserve, Ecuador. Pacific Science, 62, 191-204, 2008.

Heath, M. R.; Neat, F. C.; Pinnegar, J. K.; Reid, D. G.; Sims, D. W.; Wright, P. J. Review of climate change impacts on marine fish and shellfish around the UK and Ireland. Aquatic Conservation: Marine and Freshwater Ecosystems, 22, 337-367, 2012.

Herrmann, M.; Alfaya, J.; Lepore, M.; Penchaszadeh, P.; Arntz, W. Population structure, growth and production of the yellow clam Mesodesma mactroides (Bivalvia: Mesodesmatidae) from a high-energy, temperate beach in northern Argentina. Helgoland Marine Research, 65, 285-297, 2011.

Hilborn, R.; Orensanz, J. M.; Parma, A. Institutions, incentives and the future of fisheries. Philosophical Transactions of the Royal Society of London B, 360, 47-57, 2005.

Horta, S.; Defeo, O. The spatial dynamics of the whitemouth croaker artisanal fishery in Uruguay and interdependencies with the industrial fleet. Fisheries Research, 125-126, 121128, 2012.

Huey, R. B.; Kearney, M. R.; Krockenberger, A.; Holtum, J. A. M.; Jess, M.; Williams, S. E. Predicting organismal vulnerability to climate warming: roles of behaviour, physiology and adaptation. Philosophical Transactions of the Royal Society B, 367, 1665-1679, 2012.

Jentoft, S.; Chuenpagdee, R. Fisheries and coastal governance as a wicked problem. Marine Policy, 33, 553-560, 2009.

Jentoft, S.; Eide, A. (Eds.) Poverty mosaics: realities and prospects in small-scale fisheries. Springer Science + Business Media B.V., 2011.

Kalikoski, D. C.; Neto, P. Q.; Almudi, T. Building adaptive capacity to climate variability: the case of artisanal fisheries in the estuary of the Patos Lagoon, Brazil. Marine Policy, 34, 742-751, 2010.

Lenton, T. M. Early warning of climate tipping points. Nature Climate Change, 1, 201-209, 2011.

Ling, S. D.; Johnson, C. R.; Frusher, S. D.; Ridgway, K. R. Overfishing reduces resilience of kelp beds to climate-driven catastrophic phase shift. Proceedings of the National Academy of Sciences USA, 106, 22341-22345, 2009.

McCay, B. J.; Brandt, S.; Creed, C. F. Human dimensions of climate change and fisheries in a coupled system: the Atlantic surfclam case. ICES Journal of Marine Science, 68, 13541367, 2011.

McCay, B. J.; Jones, P. J. S. Marine Protected areas and the governance of marine ecosystems and fisheries. Conservation Biology, 25, 1130-1133, 2011.

McLachlan, A.; Dugan, J.; Defeo, O.; Ansell, A. D.; Hubbard, D. M.; Jaramillo, E.; Penchaszadeh, P. E. Beach clam fisheries. Oceanography and Marine Biology: An Annual Review, 34, 163-232, 1996.

Mellin, C.; Russell, B. D.; Connell, S. D.; Brook, B. W.; Fordham, D. A. Geographic range determinants of two commercially important marine molluscs. Diversity and Distributions, 18, 133-146, 2012.

Micheli, F.; Saenz-Arroyo, A. S.; Greenley, A.; Vazquez, L.; Espinoza-Montes, J. A.; Rossetto, M.; De Leo, G. A. Evidence that marine reserves enhance resilience to climatic impacts. PloS ONE, 7, e40832, 2012.

Mumby, P. J.; Chollett, I.; Bozec, Y-M.; Wolff, N. H. Ecological resilience, robustness and vulnerability: how do these concepts benefit ecosystem management? Current Opinion in Environmental Sustainability, 7, 22-27, 2014.

Narita, D.; Rehdanz, K.; Tol, R. S. J. Economic costs of ocean acidification: a look into the impacts on global shellfish production. Climatic Change, 113, 1049-1063, 2012. 
Orensanz, J.; Jamieson, G. S. The assessment and management of spatially structured stocks: an overview of the North Pacific Symposium on Invertebrate Stock Assessment and Management. Canadian Special Publication of Fisheries and Aquatic Sciences, 125, 441-459, 1998.

Orensanz, J.; Parma, A. M.; Jerez, G.; Barahona, N.; Montecinos, M.; Elías, I. What are the key elements for the sustainability of 'S-fisheries'? Insights from South America. Bulletin of Marine Science, 76, 527-556, 2005.

Ortega, L.; Castilla, J. C.; Espino, M.; Yamashiro, C.; Defeo, O. Large-scale and long-term effects of fishing, market price and climate on two South American sandy beach clam species. Marine Ecology Progress Series, 469, 71-85, 2012.

Ostrom, E. A general framework for analyzing sustainability of social-ecological systems. Science, 325, 419-422, 2009.

Perry, R. I.; Ommer, R. E.; Barange, M.; Jentoft, S.; Neis, B.; Sumaila, U. R. Marine social-ecological responses to environmental change and the impacts of globalization. Fish and Fisheries, 12, 427-450, 2011.

Perry, R. I.; Ommer, R. E.; Barange, M.; Werner, F. The challenge of adapting marine social-ecological systems to the additional stress of climate change Current Opinion in Environmental Sustainability, 2, 356-363, 2010.

Riascos, J. M.; Carstensen, D.; Laudien, J.; Arntz, W. E.; Oliva, M. E.; Güntner, A.; Heilmayer, O. Thriving and declining: climate variability shaping life-history and population persistence of Mesodesma donacium in the Humboldt Upwelling System. Marine Ecology Progress Series, 385, 151-163, 2009.

Rouyer, T.; Fromentin, J-M.; Ménard, F.; Cazelles, B.; Briand, K.; Pianet, R.; Planque, B.; Stenseth, N. C. Complex interplays among population dynamics, environmental forcing, and exploitation in fisheries. Proceedings of the National Academy of Sciences USA, 105, 5420-5425, 2008.
Salas, S.; Chuenpagdee, R.; Charles, A.; Seijo, J. C. (Eds.). Coastal fisheries of Latin America and the Caribbean. FAO Fisheries and Aquaculture Technical Paper N ${ }^{\circ}$ 544, Rome, 2011.

Scheffer, M.; Bascompte, J.; Brock, W. A.; Brovkin, V.; Carpenter, S. R.; Dakos, V.; Held, H.; Van Nes, E. H.; Rietkerk, M.; Sugihara, G. Early-warning signals for critical transitions. Nature, 461, 53-59, 2009.

Schoeman, D. S.; Schlacher, T. A.; Defeo, O. Climate-change impacts on sandy-beach biota: crossing a line in the sand. Global Change Biology, 2014. doi: 10.1111/gcb.12505

Stenseth, N. C.; Mysterud, A.; Ottersen, G.; Hurrell, J. W.; Chan, K-S.; Lima, M. Ecological effects of climate fluctuations. Science, 297, 1292-1296, 2002.

Taylor, M. H.; Wolff, M.; Mendo, J.; Yamashiro, C. Changes in trophic flow structure of Independence Bay (Peru) over an ENSO cycle. Progress in Oceanography, 79, 336-351, 2008.

White, C.; Costello, C. Matching spatial property rights fisheries with scales of fish dispersal. Ecological Applications, 21, 350-362, 2011.

Williams, S. E.; Shoo, L. P.; Isaac, J. L.; Hoffmann, A. A.; Langham, G. Towards an integrated framework for assessing the vulnerability of species to climate change. PLoS Biology, 6, e325, 2008.

Wolff, M.; Ruiz, D. J.; Taylor, M. El Niño induced changes to the Bolivar Channel ecosystem (Galapagos): comparing model simulations with historical biomass time series. Marine Ecology Progress Series, 448, 7-22, 2012a.

Wolff, M.; Schunauer, A.; Castrejón, M. A revised strategy for the monitoring and management of the Galapagos sea cucumber. International Journal of Tropical Biology, 60, 539-551, 2012b. 